ESAIM: PROCEEDINGS, August 2009, Vol. 28, p. 100-116

M. Ismail, B. Maury \& J.-F. Gerbeau, Editors

\title{
POLYNOMIAL LEAST-SQUARES RECONSTRUCTION FOR SEMI-LAGRANGIAN CELL-CENTERED HYDRODYNAMIC SCHEMES
}

\author{
Gilles Carré ${ }^{1}$, Stéphane Del Pino ${ }^{1}$, Kirill Pichon GostaF ${ }^{2}$, \\ Emmanuel Labourasse ${ }^{1}$ and Alexander V. Shapeev ${ }^{3}$
}

\begin{abstract}
In Inertial Confinement Fusion (ICF) simulation, use of Lagrangian hydrodynamic numerical schemes is a cornerstone. It avoids mixing of materials and allows for symmetry preservation in dimension two. Recently, [7] and then [9] proposed an interesting alternative to the historical VNR scheme [15]. These two first order schemes are multidimensional generalizations of the Godunov acoustic solver. Alternatively, a WENO Lagrangian scheme was proposed in [6]. This scheme suffers from non-preservation of symmetries and its velocity computation can be discussed.

The aim of this work is to evaluate the later scheme on ICF representative test cases and to derive a polynomial reconstruction that preserves symmetries for the three cell-centered scheme. This reconstruction is inspired by [12]. Since this paper focuses on the approximation of Euler equations, considered test cases are purely hydrodynamic and do not illustrate all difficulties encountered in ICF.

We first briefly recall different schemes used for this study. We then explain the Least-Squares ENO reconstruction that we chose for symmetry preservation and describe the limiting strategy. We finally illustrates the presented results by some representative numerical experiments.
\end{abstract}

Résumé. La simulation de Fusion par Confinement Inertiel (FCI) utilise souvent des schémas hydrodynamiques Lagrangiens. Cela permet d'éviter le mélange de matériaux et permet de préserver des symétries en dimension deux. Récemment, des alternatives intéressantes au schéma historique VNR [15] ont été proposées dans [7] puis dans [9]. Parallèlement, un schéma WENO a été proposé dans [6]. Ce schéma ne préserve pas les symétries et le calcul des vitesses peut être discuté.

L'objectif de ce travail est d'évaluer ce dernier schéma pour des cas tests représentatifs de la FCI et d'écrire une reconstruction polynomiale qui préserve les symétries pour les trois schémas étudiés. Cette reconstruction est inspirée de [12]. Puisqu'on se concentre ici sur la résolution des équations d'Euler, les cas tests présentés sont purement hydrodynamiques et ne prétendent pas couvrir l'ensemble des difficultés rencontrées en FCI.

Nous rappelons d'abord les différents schémas utilisés ici. Nous expliquons ensuite la reconstruction au sens des moindres carrés que nous avons choisie ainsi qu'une stratégie de limitation préservant aussi la symétrie. On illustre finalement les résultats au travers de quelques cas tests représentatifs choisis.

\section{INTRODUCTION}

The use of Lagrangian hydrodynamic schemes is a key ingredient for the simulation of Inertial Confinement Fusion (ICF). Practically most of the schemes are written in semi-Lagrangian coordinates - the mesh moves

\footnotetext{
${ }^{1}$ CEA, DAM, DIF, F-91297 Arpajon, France.

${ }^{2}$ Laboratoire Jacques-Louis Lions, Université Pierre et Marie Curie, 175 rue du Chevaleret, 75013 Paris.

${ }^{3}$ Department of Mathematics, National University of Singapore, 2 Science Drive 2, Singapore 117543.
}

(C) EDP Sciences, SMAI 2009 
with the flow - instead of purely Lagrangian coordinates. This paper focuses on the evaluation of high-order semi-Lagrangian schemes based on ENO-like reconstructions. Before going further, we recall the Euler equations in semi-Lagrangian coordinates.

Let $\Omega(t) \in \mathbb{R}^{2}$ a connected domain occupied by a compressible inviscid fluid at time $t$. The integral form of Euler equations in semi-Lagrangian coordinates write

$$
\begin{array}{rlrl}
d_{t} \int_{\omega(t)} 1 d \omega-\int_{\gamma(t)}(\mathbf{u}, \mathbf{n}) d \gamma & =0, & & \text { (volume conservation) } \\
d_{t} \int_{\omega(t)} \rho d \omega & =0, & & \text { (mass conservation) } \\
d_{t} \int_{\omega(t)} \rho \mathbf{u} d \omega+\int_{\gamma(t)} p \mathbf{n} d \gamma & =0, & & \text { (momentum conservation) } \\
d_{t} \int_{\omega(t)} \rho e d \omega+\int_{\gamma(t)}(p \mathbf{u}, \mathbf{n}) d \gamma & =0, &
\end{array}
$$

for any $\omega(t) \subset \Omega(t)$ and its boundary $\gamma(t)=\partial \omega(t), \mathbf{n}$ being the external normal vector to $\gamma(t)$.

In system (1-4) $\rho$ is the density, $\mathbf{u}$ is the velocity, $p=p(\rho, \epsilon)$ is the pressure and $e$ is the total energy density. We denote $\epsilon=e-\frac{1}{2} \mathbf{u} \cdot \mathbf{u}$ the internal energy density. A rigorous derivation of these equations, in a Partial Differential Equation form, can be found in [11].

A classical family of methods to solve these equations is based on multidimensional extensions of the VNR scheme [15] (see [4] for instance). All these methods use staggered discretizations in space: the velocity is defined at the nodes of the mesh, while thermodynamic quantity averages are defined in cells.

In the past years, new finite volume cell-centered schemes have been proposed. First, R. Abgrall et al proposed a Discontinuous Galerkin-like method to solve the Euler equations in Lagrangian coordinates in [2]. This inspired B. Després and C. Mazeran to develop the Glace scheme which solves the Euler equation in semi-Lagrangian coordinates [7]. Then, P.-H. Maire et al modified Glace's flux computation to build the Chic scheme which is more stable but more dissipative [9]. Alternatively, J. Cheng and C.-W. Shu published a new semi-Lagrangian scheme based on ENO reconstruction [6].

Obviously for a finite volume like discretization of system (1-4), the domain $\Omega(t)$ is replaced by a moving conformal grid $\mathcal{M}$ and $\omega(t)$ describes the cells of $\mathcal{M}$.

The aims of this study are the following:

(1) We want to evaluate the scheme proposed by J. Cheng and C.-W. Shu in the context of ICF. One of the key ingredients in ICF simulation is the ability to preserve symmetries. While this goal is not reached in [6], we achieve it in this paper.

(2) We also want to examine the behavior of ENO-like reconstruction for Glace/Chic schemes. Using these kind of schemes is of interest to us since the node velocity computed by J. Cheng and C.-W. Shu's method may break the compatibility of the gradient in divergence operators of (1-4), which is a bad property.

(3) As said previously, while the Glace scheme can be subject to lack of stability (hourglass modes), the Chic scheme suffers from being too dissipative. This can be observed in the case of isentropic convergent compressions for instance. We want to establish here the effects of using higher-order schemes to treat this problem.

This paper articulates as follows. In a first part, we briefly recall the three schemes we decided to test. Then we describe the Least-Squares ENO reconstruction that we used in order to construct higher-order versions of these schemes. This choice is discussed in the third part which is devoted to numerical experiments. Finally, we conclude and give some directions for future work.

Before going further in this paper, we need to advise the reader that high-order semi-Lagrangian schemes is a challenging topic. This is due to the fact that the equation (1) describes the evolution of the volume. So, 
a high-order description of the evolution of the geometry is mandatory to derive high-order methods - for instance one would need parabolic descriptions of the edges of the cells to get a third-order scheme. In other words we are limited to second-order of accuracy if we use polygonal cells for general flows.

However the choice of studying more than second-order - neglecting the geometry effects - is a first step. Moreover, we hope to derive better second-order schemes with this method.

\section{Cell-Centered Semi-Lagrangian schemes}

This section briefly describes the cell-centered semi-Lagrangian schemes that we considered for this study. The paragraph 1.5 describes the derivation of the higher-order schemes.

\subsection{Notations}

We use the following notations:

- $j$ denotes a cell and its number in the mesh,

- $r$ is a vertex of the mesh and its number,

- $V_{j}$ is the volume of the cell $j$ and $M_{j}$ is its mass,

- $\mathbf{x}_{r}$ denotes the coordinates of the node $r$ and $\mathbf{u}_{r}$ is its velocity,

- $\mathbf{x}_{j}$ is the center of mass of the cell $j$.

Since the finite volume schemes are cell-centered, the physical quantities are defined in cells:

- $\mathbf{u}_{j}$ is the mean of the velocity in cell $j$,

- $\rho_{j}$ denotes the mean density in cell $j$,

- $e_{j}$ and $\epsilon_{j}$ respectively represent the means of the total and internal energy densities in cell $j$,

- finally the pressure in cell $j$ is $p_{j}$, and $c_{j}$ is the local sound velocity.

\subsection{The Glace scheme}

The Glace scheme was introduced in [11] and [7]. An extension to the 3D case was given in [5]. For a more detailed and deep understanding of the scheme the reader is invited to go through the above references.

A cornerstone in the derivation of the Glace scheme is use of some special vectors defined at the vertices of each cell. These vectors, denoted $\mathbf{C}_{j r}$ and defined by

$$
\mathbf{C}_{j r}=\nabla_{\mathbf{x}_{r}} V_{j}
$$

represent the variation of the volume $V_{j}$ according to the position of the vertex $r$ of the cell $j$.

In dimension $d$, the definition (5) implies two properties linking the $\mathbf{C}_{j r}$ 's and $V_{j}$

$$
\begin{aligned}
& V_{j}=\frac{1}{d} \sum_{r}\left(\mathbf{C}_{j r}, \mathbf{x}_{r}\right), \\
& d_{t} V_{j}=\sum_{r}\left(\mathbf{C}_{j r}, \mathbf{u}_{r}\right) .
\end{aligned}
$$

Considering a mesh of the computational domain, we now describe the semi-discrete scheme (continuous in time).

The relation (7) is an exact discretization of equation (1) for any cell $j$ defined by the set of its control vertices $r$. This gives the approximation of $\int_{\partial j}(\mathbf{u}, \mathbf{n})=\int_{j} \nabla \cdot \mathbf{u} \approx \sum_{r}\left(\mathbf{C}_{j r}, \mathbf{u}_{r}\right)$.

Building a compatible scheme (discrete divergence operator being the adjoint of the discrete gradient), the following approximations are used:

$$
\int_{\partial j} p \mathbf{n}=\int_{j} \nabla p \approx \sum_{r} \mathbf{C}_{j r} p_{r}
$$


and

$$
\int_{\partial j} p \mathbf{u} \cdot \mathbf{n}=\int_{j} \nabla \cdot(p \mathbf{u}) \approx \sum_{r}\left(\mathbf{C}_{j r}, p_{r} \mathbf{u}_{\mathbf{r}}\right)
$$

This leads to the semi-discrete in space finite volume scheme structure:

$$
\begin{aligned}
d_{t} V_{j}(t) & =\sum_{r}\left(\mathbf{C}_{j r}, \mathbf{u}_{r}\right), \\
d_{t} \int_{j} \rho_{j}(t) & =0 \\
d_{t} \int_{j} \rho_{j}(t) \mathbf{u}_{j}(t) & =-\sum_{r} \mathbf{C}_{j r} p_{r}, \\
d_{t} \int_{j} \rho_{j}(t) e_{j}(t) & =-\sum_{r}\left(\mathbf{C}_{j r}, p_{r} \mathbf{u}_{r}\right) .
\end{aligned}
$$

An Euler explicit scheme can be used to perform the time discretization. It only remains to compute $\mathbf{u}_{r}$ and $p_{r}$ in order to write down the scheme. Three hypothesis are proposed to solve this problem:

- $p_{r}$ is replaced by some $p_{j r}$, this means that at vertices, pressures imposed by cells may be different.

- Local conservation - around a vertex — is imposed thanks to the relation

$$
\sum_{j} \mathbf{C}_{j r} p_{j r}=\mathbf{0}
$$

- The acoustic Riemann invariant relation $d p+\rho c d \mathbf{u} \cdot \mathbf{n}=0$ is chosen to link $u_{r}, p_{j r}$ and the cell-centered quantities:

where $\mathbf{n}_{j r}=\frac{\mathbf{C}_{j r}}{\left\|\mathbf{C}_{j r}\right\|}$.

$$
p_{j r}-p_{j}+\rho_{j} c_{j}\left(\mathbf{u}_{r}-\mathbf{u}_{j}, \mathbf{n}_{j r}\right)=0
$$

These hypothesis are enough to compute the fluxes $\mathbf{u}_{r}$ and $p_{j r}$ 's according to the cell-centered values and then to compute the unknowns using (8-11). The idea is to multiply (13) by $\mathbf{C}_{j r}$ and sum over the cells surrounding the vertex $r$. One then gets the linear equation on $\mathbf{u}_{r}$ :

$$
\left(\sum_{j} \rho_{j} c_{j} \frac{\mathbf{C}_{j r} \otimes \mathbf{C}_{j r}}{\left\|\mathbf{C}_{j r}\right\|}\right) \mathbf{u}_{r}=\sum_{j}\left(\rho_{j} c_{j} \frac{\mathbf{C}_{j r} \otimes \mathbf{C}_{j r}}{\left\|\mathbf{C}_{j r}\right\|} u_{j}+\mathbf{C}_{j r} p_{j r}\right),
$$

which is well-posed. Then $p_{j r}$ 's are deduced from (13).

The scheme finally writes:

- Assume that at time step $n$ all physical and geometric quantities are known.

- Compute $\mathbf{u}_{r}^{n}$ and $p_{j r}^{n}$ as described above.

- Update velocity and total energy inside cells:

$$
\begin{aligned}
& M_{j} \frac{\mathbf{u}_{j}^{n+1}-\mathbf{u}_{j}^{n}}{\Delta t}=-\sum_{r} \mathbf{C}_{j r}^{n} p_{j r}^{n}, \\
& M_{j} \frac{e_{j}^{n+1}-e_{j}^{n}}{\Delta t}=-\sum_{r}\left(\mathbf{C}_{j r}^{n}, p_{j r}^{n} \mathbf{u}_{r}^{n}\right) .
\end{aligned}
$$

- Move the mesh

and recompute $\mathbf{C}_{j r}$ 's.

$$
\mathbf{x}_{r}^{n+1}=\mathbf{x}_{r}^{n}+\Delta t \mathbf{u}_{r}^{n}
$$


- Compute the new cell volumes

$$
V_{j}^{n+1}=\frac{1}{2} \sum_{r}\left(\mathbf{C}_{j r}^{n+1}, \mathbf{x}_{r}^{n+1}\right) .
$$

- Finally update the density

$$
\rho_{j}^{n+1}=\frac{M_{j}}{V_{j}^{n+1}} .
$$

This scheme is shown to be conservative in total energy, entropic and consistent [7].

\subsection{The Chic scheme}

The Chic scheme is a variant of the Glace scheme to achieve some properties. The scheme has been proposed in [9] to degenerate exactly to the acoustic Godunov scheme for $1 \mathrm{D}$ problems. So, it is not sensitive to the aspect ratio of the cells for multidimensional meshes. For a detailed description of the scheme refer to [9] or to [10] for its 3D extension.

In fact, to build it, the Glace hypothesis are just slightly changed. The first ingredient consists in decomposing the nodal normal vector into two parts: the length of two half edges arising from the vertex times the associated outgoing normal vectors. This introduces the vectors $\mathbf{N}_{j r}^{ \pm}$defined by

$$
\mathbf{N}_{j r}^{-}=\frac{1}{2}\left\|\mathbf{x}_{r}-\mathbf{x}_{r-1}\right\| \mathbf{n}_{j r}^{-} \quad \text { and } \quad \mathbf{N}_{j r}^{+}=\frac{1}{2}\left\|\mathbf{x}_{r+1}-\mathbf{x}_{r}\right\| \mathbf{n}_{j r}^{+}
$$

where $\mathbf{n}_{j r}^{-}$and $\mathbf{n}_{j r}^{+}$are respectively the outgoing normals to cell $j$ of edges $\left(\mathbf{x}_{r}, \mathbf{x}_{r-1}\right)$ and $\left(\mathbf{x}_{r+1}, \mathbf{x}_{r}\right)$, and $r$ denotes a local numbering of vertices.

The hypothesis for the Chic scheme are the following:

- $p_{r}$ is replaced by some $p_{j r}^{ \pm}$, this means that at half-edges, pressures imposed by cells may be different.

- The local conservation now writes

$$
\sum_{j} \mathbf{N}_{j r}^{-} p_{j r}^{-}+\mathbf{N}_{j r}^{+} p_{j r}^{+}=\mathbf{0}
$$

- The acoustic Riemann invariant $d p+\rho c d \mathbf{u} \cdot \mathbf{n}=0$ is imposed for each pressure:

$$
\begin{aligned}
& p_{j r}^{-}-p_{j}+\rho_{j} c_{j}\left(\mathbf{u}_{r}-\mathbf{u}_{j}, \mathbf{n}_{j r}^{-}\right)=0 \\
& p_{j r}^{+}-p_{j}+\rho_{j} c_{j}\left(\mathbf{u}_{r}-\mathbf{u}_{j}, \mathbf{n}_{j r}^{+}\right)=0 .
\end{aligned}
$$

Then the resolution of $p_{j r}^{ \pm}$'s and $\mathbf{u}_{r}$ proceeds the same way as previously. The scheme is also consistent, entropic and conservative in total energy [9].

\subsection{The Cheng and Shu's scheme}

In the same paper as for the ENO extension [6], J. Cheng and C.-W. Shu proposed a class of semi-Lagrangian schemes for 2D simulations based on well-known 1D-fluxes, namely the Godunov flux, the Dukowicz flux, the Lax-Friedrichs flux and the HLLC flux. The general feature of this class of schemes is to compute the 1Dfluxes at the location of the quadrature points used for the integration of the fluxes on the edges. For the first order scheme, the quadrature points are simply located at the center of the edges. In this work we have chosen to calculate the fluxes thanks to an acoustic Godunov scheme for two reasons: 1- this leads to a real semi-Lagrangian scheme, meaning that the mass fluxes are zero $^{1}, 2$ - this is coherent with the computation of the fluxes for the two previously described schemes.

\footnotetext{
${ }^{1}$ It is not the case for all the fluxes proposed in [6].
} 
This leads to the following spatial discretization:

$$
\begin{aligned}
d_{t} \int_{j} \rho_{j}(t) & =0 \\
d_{t} \int_{j} \rho_{j}(t) \mathbf{u}_{j}(t) & =-\sum_{e} \mathbf{n}_{e} p_{e} l_{e}, \\
d_{t} \int_{j} \rho_{j}(t) e_{j}(t) & =-\sum_{e} p_{e} u_{e} l_{e}
\end{aligned}
$$

where the subscript $e$ stands for the edges of the cell $j, \mathbf{n}_{e}$ stands for the outgoing normal to the edge $e, l_{e}$ is the length of the edge, and $p_{e}$ and $u_{e}$ are the pressure and the normal velocity calculated at the edge. These two last quantities are obtained using the 1D acoustic Godunov solver

$$
\begin{aligned}
& p_{e}=\frac{1}{2}\left(p_{j+}+p_{j-}\right)-\frac{\rho c_{e}}{2}\left(\mathbf{u}_{j+}-\mathbf{u}_{j-}, \mathbf{n}_{e-+}\right), \\
& u_{e}=\frac{1}{2}\left(\mathbf{u}_{j+}+\mathbf{u}_{j-}, \mathbf{n}_{e-+}\right)-\frac{1}{2 \rho c_{e}}\left(p_{j+}-p_{j-}\right),
\end{aligned}
$$

with the subscripts $j+$ and $j$ - accounting for the right and left cells according to the direction of the normal to the edge $\mathbf{n}_{e-+}$, and $\rho c_{e}$ being an evaluation of the acoustic impedance at the edge. In this work, we take $\rho c_{e}=\frac{1}{2}\left(\rho_{j+} c_{j+}+\rho_{j-} c_{j-}\right)$.

It is worth noting that the velocity of the nodes is not a partial result of the computation of the fluxes, as for the two precedent schemes. J. Cheng and C.-W. Shu proposed to calculate it the following way, for a $2 \mathrm{D}$ Cartesian grid:

$$
\mathbf{u}_{r}=\frac{1}{4} \sum_{e}\left(u_{e} \mathbf{n}_{e-+}+w_{e} \mathbf{t}_{e}\right)
$$

where $\mathbf{t}_{e}$ stands for the direction of the edge.

The tangential to the edge counterpart $w_{e}$ is obtained in the following way:

$$
w_{e}=\frac{1}{2}\left(\mathbf{u}_{j+}+\mathbf{u}_{j-}, \mathbf{t}_{e}\right)
$$

There is no reason for this calculation of the velocity to lead to a compatible scheme. Let us recall that the relation (8) still holds, since it is a purely geometrical result. It implies a definition of the divergence operator, which is in general different from the one involved by the equation (20), i.e.

$$
\sum_{e} u_{e} l_{e} \neq \sum_{r}\left(\mathbf{C}_{j r}, \mathbf{u}_{r}\right)
$$

This is obvious considering that the calculation of the right term involves 9 cells for a $2 \mathrm{D}$ structured grid, whereas the calculation of the left one takes into account only 5 cells.

\subsection{High-order versions of semi-Lagrangian schemes}

For all previously described first-order schemes, we consider the same order increasing strategy. The time order is obtained by Runge-Kutta methods and the space order consists in polynomial reconstructions that are used for flux computations instead of cell mean values.

For instance, in the Glace scheme, it just consist in replacing (13) by

$$
p_{j r}-\overline{p_{j}}\left(\mathbf{x}_{r}\right)+\rho_{j} c_{j}\left(\mathbf{u}_{r}-\overline{\mathbf{u}_{j}}\left(\mathbf{x}_{r}\right), \mathbf{n}_{j r}\right)=0
$$

where $\overline{p_{j}}$ and $\overline{\mathbf{u}_{j}}$ are the reconstructed values of the pressure and velocity in the cell $j$. 
However, in (8-11) high-order fluxes are used to compute the conservative quantities $\rho_{j} \mathbf{u}_{j}$ and $\rho_{j} e_{j}$. Thus, these are the right quantities to reconstruct. So, to achieve high-order, we compute the polynomial reconstructions $\overline{\rho_{j}}, \overline{(\rho \mathbf{u})_{j}}$ and $\overline{(\rho e)_{j}}$, then we deduce

$$
\overline{\mathbf{u}_{j}}=\frac{\overline{(\rho \mathbf{u})_{j}}}{\overline{\rho_{j}}}, \quad \overline{\epsilon_{j}}=\frac{\overline{(\rho e)_{j}}-\frac{1}{2} \frac{\overline{(\rho \mathbf{u})_{j}} \cdot \overline{(\rho \mathbf{u})_{j}}}{\overline{\rho_{j}}}}{\overline{\rho_{j}}}, \quad \overline{p_{j}}=p\left(\overline{\rho_{j}}, \overline{\epsilon_{j}}\right) .
$$

Results presented in section 3.1.1 validates this choice — see right part of figure 3 .

Remark 1.1. (Flux integration) In order to achieve a high-order scheme, fluxes should also be integrated with an appropriate quadrature formula. In fact the quadrature formula used in (8), (10) and (11) is exact for affine functions along edges. Thus, we do not obtain order higher than 2.

\section{Least-Squares ENO RECONSTRUCTION}

The aim of the study is to evaluate ENO-like semi-Lagrangian schemes in the context of Inertial Confinement Fusion (ICF) type flows.

The first remark concerning this strategy is about the symmetry preservation which is a key ingredient for ICF-like simulations. This constraint cannot be satisfied by ENO reconstructions as described in [1].

For instance, assume a 1D radial simulation such as the one described in section 3.1.3. Many stencils provide polynomials with the same oscillations that are not discriminated by the ENO reconstruction. Each of them leads to a different reconstruction but they are not radial reconstruction and the symmetry is lost.

On the other hand, in [6], symmetry preservation is not achieved. The authors do not specify the reason for it: whether it is due to boundary conditions or to the WENO reconstruction scheme itself.

These considerations led us to look at the Least-Squares ENO reconstruction proposed in [12] and [13]. Obviously, this kind of polynomial reconstruction preserves symmetries. We present here a slightly modified version of this method.

\subsection{Reconstruction of smooth functions}

Let $q$ be a piecewise constant function defined on a conformal mesh $\mathcal{M}$. In each cell $j$ of $\mathcal{M}$ the value of $q$ is $q_{j}$. In this section, we compute a Least-Squares polynomial conservative reconstruction of $q$.

\subsubsection{Polynomial basis}

We consider a polynomial basis $\mathcal{B}^{j}=\left\{e_{k}^{j}\right\}_{k \geq 0}$ in cell $j$ such that $\int_{j} e_{k}^{j}=0$ for $k>0$. Recalling that $\mathbf{x}_{j}$ denotes the center of mass of cell $j$, the polynomial basis that we consider is derived from the canonical basis $\mathcal{B}=\left\{e_{k}\right\}_{k \geq 0}$ in the following way:

$$
\mathcal{B}^{j}=\left\{1, \quad e_{1}\left(\cdot-\mathbf{x}_{j}\right)-\frac{1}{V_{j}} \int_{j} e_{1}\left(\cdot-\mathbf{x}_{j}\right), \quad e_{2}\left(\cdot-\mathbf{x}_{j}\right)-\frac{1}{V_{j}} \int_{j} e_{2}\left(\cdot-\mathbf{x}_{j}\right), \ldots\right\},
$$

in other words $e_{0}^{j}=1$ and $e_{k}^{j}=e_{k}\left(\cdot-\mathbf{x}_{j}\right)-\frac{1}{V_{j}} \int_{j} e_{k}\left(\cdot-\mathbf{x}_{j}\right)$ for $k>0$.

In this paper we keep the opportunity to choose either $\mathbb{Q}^{N}$, the set of polynomial of maximum degree $N$ in each variable, or $\mathbb{P}^{N}$, the set of polynomial of maximum total degree $N$, as defined in the finite element context. This choice does not affect the following discussion.

The reconstructed polynomial $P_{j} \in \mathcal{B}^{j}$ for $q$ writes

$$
P_{j}=q_{j}+\sum_{k \geq 1}^{n_{\mathcal{B}^{j}}} p_{k}^{j} e_{k}^{j}, \quad \text { where } p_{k}^{j} \in \mathbb{R}
$$

By construction, one has $\frac{1}{V_{j}} \int_{j} P_{j}=q_{j}$, since $\int_{j} e_{k}^{j}=0$ for $k>0$, which is conservative. 


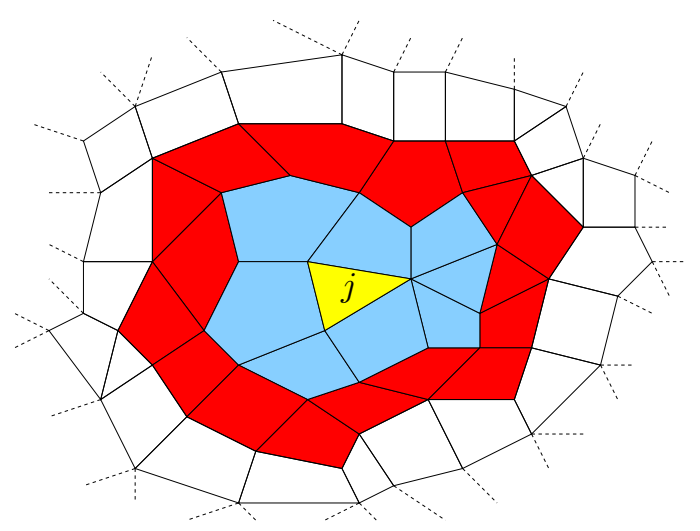

Figure 1. Stencil construction for polynomial reconstruction of degree 2 in cell $j$. Blue cells are level 1 cells, red cells are level 2 cells. The stencil $S^{j}$ is composed of blue and red cells.

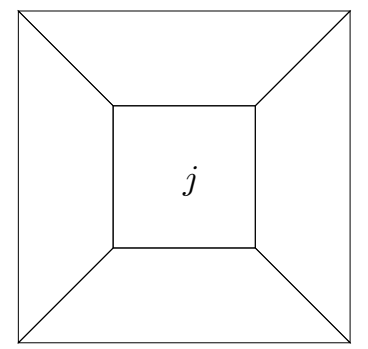

FiguRE 2. An example of underdetermined least-squares problem: no constraint on the term $x y$ is imposed for reconstruction of a $\mathbb{Q}^{1}$ function using the stencil $S^{j}$.

\subsubsection{Stencil consideration}

In order to locally reconstruct the polynomial $P_{j}$, we need to use the stencil of neighbors of the cell $j$ defined as follows:

- the set of level 0 neighbors are the cell $j$ itself, which are not a part of the stencil,

- the set of level $k$ neighbors is the set of cells that share a vertex with cells of level $k-1$ which do not belong to level $k-1$.

The considered stencil is the union of the cells of levels 1 to $N$, for the reconstruction of a polynomial of degree $N$ - see Figure 1 . The stencil associated to the cell $j$ is denoted by $S_{j}$.

However, we previously insisted on the importance of the symmetry preservation for ICF-like computations. So, the construction of $N$ layers of symmetric ghost-cells is mandatory for boundary conditions of symmetry. These ghost cells are only used for the reconstruction.

\subsubsection{Least-Squares problem}

Since we do a degree $N$ conservative reconstruction, in dimension 2 the number of unknowns is $N^{2}-1$ for a $\mathbb{Q}^{N}$ reconstruction and $N(N+1) / 2-1$ for a $\mathbb{P}^{N}$ reconstruction — see equation (26). At the same time, for a Cartesian grid, the stencil is composed of $(2 N+1)^{2}-1$ cells. So, the linear system to compute $P_{j}$ is overdetermined as we will see now. This implies to search for a least-squares solution of this problem.

In what follows, we denote by $n_{\mathcal{B}^{j}}$ the number of polynomials of degree greater than 1 of $\mathcal{B}^{j}$, and $n_{S^{j}}$ the number of cells of the stencil $S^{j}$ — keep in mind that the cell $j$ itself is not a member of $S^{j}$. 
Remark 2.1. It may occur that the linear system is underdetermined. This can be the case even if the number of stencil cells is bigger than the number of degrees of freedom in a multidimensional context - see Figure 2. If this situation is encountered, it is enough to lower locally the degree of the polynomial.

In the ideal case of $q$ being the projection - in the finite volume sense - of a polynomial of degree $N$ the reconstruction must be exact, so it is natural to impose for any cell $i$ of the stencil $S_{j}$

$$
\frac{1}{V_{i}} \int_{i} P_{j}(\mathbf{x})=q_{i}
$$

According to the equation (26), (27) writes

$$
\frac{1}{V_{i}} \sum_{k=1}^{n_{\mathcal{B} j}} \int_{i} e_{k}^{j}(\mathbf{x}) d \mathbf{x} p_{k}^{j}=q_{i}-q_{j} .
$$

These equations form the linear problem

$$
A^{j} U^{j}=B^{j}
$$

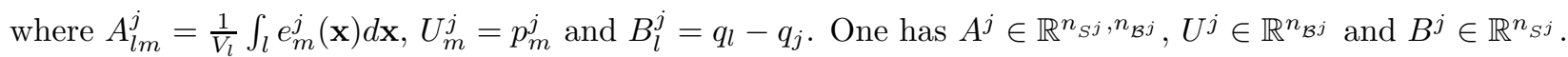

To solve this least-squares problem, one can use many methods. We chose two algorithms: Householder and Givens rotations - see [8] for instance. Both of these algorithms are able to detect underdetermined systems, so we can detect while solving if the stencil must be lowered (or alternatively if the polynomial degree must be decreased). An interesting fact is that even if the complexity of Householder method is smaller than Givens: respectively $2 n_{\mathcal{B}^{j}}^{2}\left(n_{S^{j}}-\frac{1}{3} n_{\mathcal{B}^{j}}\right)$ and $3 n_{\mathcal{B}^{j}}^{2}\left(n_{S^{j}}-\frac{1}{3} n_{\mathcal{B}^{j}}\right)$ flops, we experimentally established that Givens method was faster! ${ }^{2}$

Finally, one can note that since both of these algorithms lead to a $Q R$ factorization, the same factorization can be used to perform the smooth reconstruction of all variables at once. This is important with regard to an efficient implementation of the method.

\subsubsection{Condition number of the Least-Squares Problem.}

In [1], R. Abgrall showed the influence of the chosen polynomial basis on the condition number of the leastsquares matrix. He proposed using of the following barycentric coordinates basis: for a triangle $j$ he took the polynomial basis $\left\{\lambda_{1}^{l} \lambda_{2}^{k}\right\}_{k+l \leq N}$ where $\lambda_{1}+\lambda_{2}+\lambda_{3}=1$ are barycentric coordinates in cell $j$.

This has the effect of normalizing the columns of the least-squares problem which leads to a much better condition number that improves the quality of the approximation. In one dimension of space this is equivalent to replacing the basis vector $e_{k}^{j}=\left(x-x_{j}\right)^{k}$ by $\left(\frac{x-x_{r}}{x_{r+1}-x_{r}}\right)^{k}$ for instance. The normalization effect comes from the denominator term $V_{j}^{k}=\left(x_{r+1}-x_{r}\right)^{k}$. For multidimensional problems using non-simplexes cells, this kind of normalization can be approximated by replacing $e_{k}^{j}$ with $\left(\frac{1}{V_{j}}\right)^{\frac{d_{k}}{d}} e_{k}^{j}$, where $d_{k}$ is the total degree of $e_{k}^{j}$, and $d$ the space dimension. Reinterpreting this scaling as a column-weighting strategy, one can rewrite the problem (29) as

where $G^{j}=\operatorname{diag}\left(\left(\frac{1}{V_{j}}\right)^{\frac{d_{1}}{d}},\left(\frac{1}{V_{j}}\right)^{\frac{d_{2}}{d}}, \ldots\right) \in \mathbb{R}^{n_{\mathcal{B}^{j}}, n_{\mathcal{B}^{j}}}$.

$$
A^{j} G^{j} Y^{j}=F^{j}, \quad \text { and } \quad U^{j}=G^{j} Y^{j},
$$

However, as mentioned in [8] (paragraph 5.6.1), there exists a quasi-optimal choice of a matrix $G^{j}$ that approximately minimizes the condition number of $A^{j} G^{j}$. Denoting $A^{j}(:, l)$ to be the column vector $l$ of $A^{j}$ and $\|\cdot\|_{2}$ to be the $l^{2}$ norm, it has been shown in [14] that

$$
G^{j}=\operatorname{diag}\left(\frac{1}{\left\|A^{j}(:, 1)\right\|_{2}}, \frac{1}{\left\|A^{j}(:, 2)\right\|_{2}}, \ldots\right),
$$

\footnotetext{
${ }^{2}$ This might be due to the column pivoting procedure used in the Householder algorithm: these operations are not taken into account in the complexity of the algorithm.
} 
is such a matrix. This is the column-weighting that we used in this work.

In this section, we have described an order $N+1$ reconstruction method for smooth functions that preserves symmetries by construction. However, the aim of this work is to approximate Euler equations. We cannot use this strategy as is for non-regular functions.

We now describe the technique used in [12] and then propose a simple limitation strategy that preserves symmetries. This second strategy can no longer be considered as ENO since the method is not uniformly of order $N+1$ as expected for an ENO-like reconstruction of degree $N$.

\subsection{Least-Squares ENO Data-Dependent reconstruction}

For a detailed description of the method, refer to [12]. In this paper, a row-weighting is proposed to take into account the distance of the cells of the stencil $S_{j}$ from the cell $j$. The proposed row-weighted system to solve is

$$
W^{j} A^{j} U^{j}=W^{j} B^{j}
$$

where

$$
W^{j}=\operatorname{diag}\left(\frac{1}{\left\|\mathbf{x}_{j}-\mathbf{x}_{S_{j}^{1}}\right\|}, \frac{1}{\left\|\mathbf{x}_{j}-\mathbf{x}_{S_{j}^{2}}\right\|}, \ldots\right) \in \mathbb{R}^{n_{S^{j}}, n_{S^{j}}},
$$

where $S_{j}^{i}$ is the $i^{\text {th }}$ cell of the stencil $S_{j}$. Again, the reader is invited to read the paragraph 5.6.2 of [8] for a discussion about row-weighting.

In this case, the column-weighting proposed in (31) is computed on the matrix $W^{j} A^{j}$. Note that to simplify notations, column-weighting is omitted in what follows.

Using this row-weighting, the approximation order in unchanged. As previously, oscillations may arise from the reconstruction of non-smooth functions.

In order to cure this behavior for non-smooth data reconstruction, row-weighting is also used. The idea is to use very small weights for rows associated to cells where a discontinuity is detected.

Let $Q^{j} \in \mathbb{R}^{n_{S^{j}}, n_{S^{j}}}$ and $R^{j} \in \mathbb{R}^{n_{S^{j}}, n_{\mathcal{B}^{j}}}$ the matrices of the $Q R$-factorization of $W^{j} A^{j}$. One has $Q^{j^{T}} Q^{j}=I$ and

$$
R^{j}=\left(\begin{array}{ccc}
\times & \cdots & \times \\
0 & \ddots & \vdots \\
0 & \cdots & \times \\
0 & \cdots & 0 \\
\vdots & & \vdots \\
0 & \cdots & 0
\end{array}\right),
$$

is a upper triangular matrix. Let $\mathcal{R}^{j}=Q^{j^{T}} W^{j} B^{j}$, the least-squares problem now writes $R^{j} U^{j}=\mathcal{R}^{j}$.

If the polynomial reconstruction is exact - the data function $q$ is the projection in the finite volume sense of a polynomial of degree lower than $N$ - the values $\mathcal{R}_{k}^{j}=0 \forall k$ such that $n_{\mathcal{B}^{j}}<k \leq n_{S^{j}}$.

If the reconstruction is not exact, these values are non-zeros and the last $n_{S^{j}}-n_{\mathcal{B}^{j}}$ equations are not verified.

This is why it is proposed in [12] to use the residual $\left(\sum_{k=n_{\mathcal{B}^{j}}+1}^{n_{S^{j}}} \mathcal{R}_{k}^{j}\right)^{\frac{1}{2}}$ as an indicator. In fact, to get rid of the geometrical mesh scale effects, the indicator is finally

$$
r=\left(n_{S^{j}} \frac{\sum_{k=n_{\mathcal{B}^{j}}+1}^{n_{S^{j}}} \mathcal{R}_{k}^{j}}{\sum_{k=1}^{n_{S^{j}}} W_{k}^{j^{2}}}\right)^{\frac{1}{2}} .
$$


This allows to define a new weighting that takes into account the smoothness of the data to reconstruct. We write it as a data-dependent diagonal matrix:

$$
D^{j}=\operatorname{diag}\left(\frac{1}{1+\kappa r\left|\frac{q_{j}-q_{S_{1}^{j}}}{\left\|\mathbf{x}_{j}-\mathbf{x}_{S_{1}^{j}}\right\|}\right|^{\kappa+1}}, \frac{1}{1+\kappa r\left|\frac{q_{j}-q_{S_{2}^{j}}}{\left\|\mathbf{x}_{j}-\mathbf{x}_{S_{2}^{j}}\right\|}\right|^{\kappa+1}}, \ldots\right) \in \mathbb{R}^{n_{S^{j}}, n_{S^{j}}},
$$

where $\kappa$ is a scalar, for which a good value seems to be $k=0.1$. In [12], C.F. Ollivier-Gooch then discusses the values of $D_{k k}^{j}$, using the following cut-off. If $r\left|\frac{q_{j}-q_{S_{i}^{j}}}{\left\|\mathbf{x}_{j}-\mathbf{x}_{S_{i}^{j}}\right\|}\right|^{\kappa+1}>0.1$ the data is considered as non-smooth and the stencil can be modified by removing the cell $i$. If the stencil becomes too small, the degree of the polynomial reconstruction has to be lowered. In this study we did not try this option. We used the data-dependent weighting of the initial stencil, solving

$$
D^{j} W^{j} A^{j} U^{j}=D^{j} W^{j} B^{j}
$$

Remark 2.2. This strategy is not compatible with (25) kind of reconstruction. Thus, one has to reconstruct $p_{j}$ and $\mathbf{u}_{j}$ fields. However we think that this is not the right choice for a high-order reconstruction as described in section 1.5 .

\subsection{Limitation strategy}

In this section, we present a simple limiter strategy designed to preserve symmetries and to be used conjointly with the reconstruction of conservative variables leading to the computation of $\overline{\mathbf{u}_{j}}$ and $\overline{p_{j}}$ defined in (25).

\subsubsection{Scalar quantities}

We consider the set $\mathcal{C}$ of necessary cells for a given first-order flux computation. We denote $q^{\max }=\max _{j \in \mathcal{C}} q_{j}$ and $q^{\min }=\min _{j \in \mathcal{C}} q_{j}$. The limiter strategy is very simple: let $\xi$ be a quadrature vertex of the flux computation, and $P_{j}$ be the polynomial reconstruction in the cell $j \in \mathcal{C}$. At any quadrature vertex $\xi$, the limited reconstructed value $\overline{P_{j}}$ is defined as follows:

- if $P_{j}(\xi)<q^{\text {min }}$ then $\overline{P_{j}}=q^{\text {min }}$,

- if $q^{\min } \leq P_{j}(\xi) \leq q^{\max }$ then ${\overline{P_{j}}}_{\xi}=P_{j}(\xi)$,

- if $q^{\max }<P_{j}(\xi)$ then $\overline{P_{j}}=q^{\max }$.

\subsubsection{Vector limitation}

Vector quantities could be limited using the same technique component by component. However, this strategy is prohibited by the objective of symmetry preservation, especially in the case of convergent flows.

The idea is then to base the limiter on the norm of the vector.

We keep the same notations as previously. Let the $\mathbb{R}^{2}$ piecewise constant function $\mathbf{q}$ of components $q^{1}$ and $q^{2}$. Let $P_{j}^{1}$ and $P_{j}^{2}$ their respective reconstructions in a cell $j \in \mathcal{C}$. Let $\mathbf{P}_{j}=\left(P_{j}^{1}, P_{j}^{2}\right)$. We now define $q^{\max }=\max _{j \in \mathcal{C}}\left\|\mathbf{q}_{j}\right\|$ and $q^{\min }=\min _{j \in \mathcal{C}}\left\|\mathbf{q}_{j}\right\|$. The reconstructed limited value $\overline{\mathbf{P}}_{j}$ at any quadrature vertex $\xi$ is given by

- if $\left\|\mathbf{P}_{j}(\xi)\right\|<q^{\min }$ then $\overline{\mathbf{P}}_{j}=\frac{q^{\min }}{\left\|\mathbf{P}_{j}(\xi)\right\|} \mathbf{P}_{j}(\xi)$,

- if $q^{\min } \leq\left\|\mathbf{P}_{j}(\xi)\right\| \leq q^{\max }$ then $\overline{\mathbf{P}}_{j}=\mathbf{P}_{j}(\xi)$,

- if $q^{\max }<\left\|\mathbf{P}_{j}(\xi)\right\|$ then $\overline{\mathbf{P}_{j}}=\frac{q^{\max }}{\left\|\mathbf{P}_{j}(\xi)\right\|} \mathbf{P}_{j}(\xi)$. 


\section{Numerical tests}

Numerical tests presented in this paper are performed with the G0++ code by P. Hoch [3].

\subsection{Smooth cases}

This section begins with the Kidder isentropic compression test case in both $1 \mathrm{D}$ and $2 \mathrm{D}$. The $2 \mathrm{D}$ case is an isentropic convergent problem. Since the solution is smooth, we show the accuracy of the polynomial reconstruction we proposed on this particular problem. The 1D problem preserves polygonal cells - straight edges remain straight during the compression. Moreover, in this particular case, the quadrature formula is exact — remark 1.1 does not hold. For this case, the resolution of equation (1) is of the correct order.

\subsubsection{D Kidder isentropic compression}

The analytical solution is the following

$$
\begin{aligned}
\rho(x, t) & =\frac{\left(\frac{\rho_{2}^{\gamma-1}\left(\frac{x^{2}}{h(t)^{2}}-R_{1}^{2}\right)+\rho_{1}^{\gamma-1}\left(R_{2}^{2}-\frac{x^{2}}{h(t)^{2}}\right)}{R_{2}^{2}-R_{1}^{2}}\right)^{\frac{1}{\gamma-1}}}{h(t)}, \\
u(x, t) & =-\frac{x t}{1-t^{2}}, \\
\epsilon(x, t) & =\rho(x, t)^{\gamma-1}, \\
p(x, t) & =(\gamma-1) \rho(x, t)^{\gamma},
\end{aligned}
$$

with $\gamma=3, h(t)=\sqrt{1-\frac{t^{2}}{\tau^{2}}}$ and $\tau=\sqrt{\frac{1}{2 \gamma} \frac{R_{2}^{2}-R_{1}^{2}}{\rho_{2}^{\gamma-1}-\rho_{1}^{\gamma-1}}}$. For the presented results, we took $\rho_{1}=1, \rho_{2}=2, R_{1}=0$ and $R_{2}=10$. The final time is $t=1.2$.

Computations are performed in $2 \mathrm{D} i$
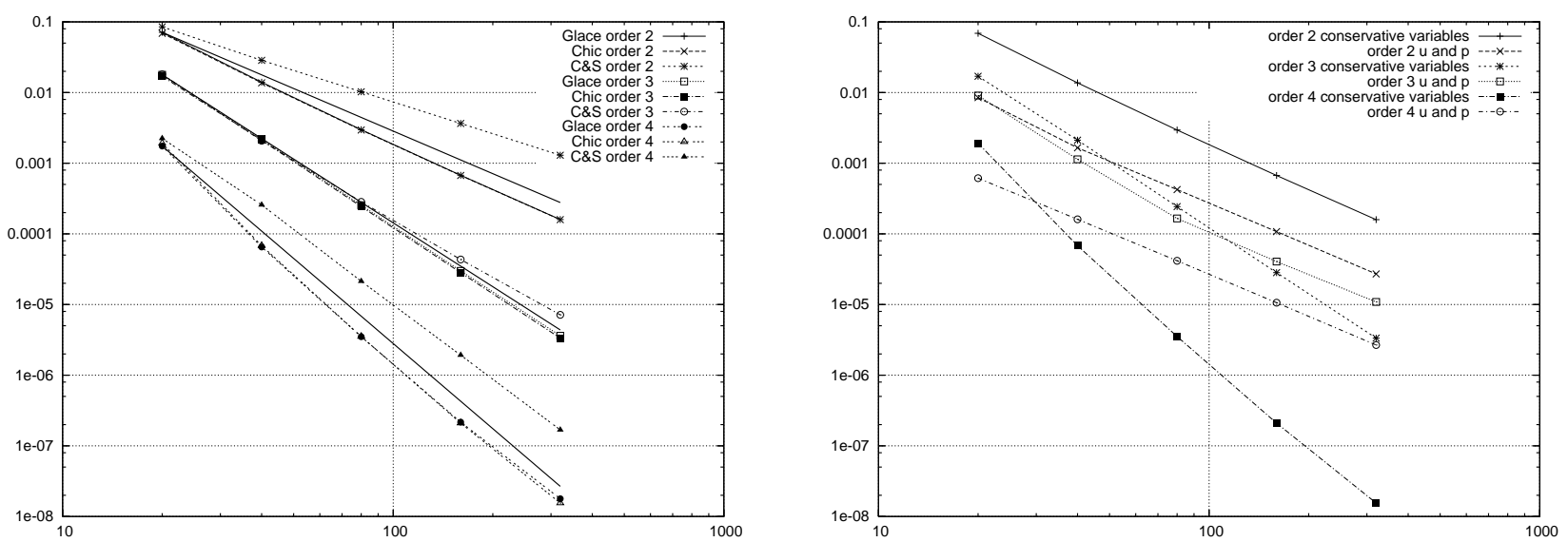

Figure 3. Convergences in log scale. $L^{2}$-norm of the error for the density against the number of cells in the $x$-direction. Left: expected convergence rates are obtained for all schemes using conservative variables reconstruction (25). Bold lines represent theoritical orders. Right: reconstruction of non-conservative variables $\mathbf{u}$ and $p$ makes the scheme fall to order 2 - here Chic is used. 
The figure 3 shows the expected behavior of the polynomial reconstruction for the density: the expected orders are achieved. Note that these results are obtained while the limiter described in 2.3 is activated. This indicated its suitability in smooth regions.

The right part illustrates the loss of accuracy if the non-conservative variables $\mathbf{u}$ and $p$ are directly reconstructed as described in section 1.5. These poor results are obtained without limitation which is thus not the cause of this loss of accuracy. We give these results using the Chic scheme as an example.

Same results are obtained for momentum and total energy.

\subsubsection{D Kidder isentropic compression}

In dimension 2 the solution becomes

$$
\begin{aligned}
\rho(\mathbf{x}, t) & =\frac{\left(\frac{\rho_{2}^{\gamma-1}\left(\frac{\mathbf{x} \cdot \mathbf{x}}{h(t)^{2}}-R_{1}^{2}\right)+\rho_{1}^{\gamma-1}\left(R_{2}^{2}-\frac{\mathbf{x} \cdot \mathbf{x}}{h(t)^{2}}\right)}{R_{2}^{2}-R_{1}^{2}}\right)^{\frac{1}{\gamma-1}}}{h(t)^{2}}, \\
\mathbf{u}(\mathbf{x}, t) & =-\frac{\mathbf{x} t}{1-t^{2}}, \\
\epsilon(\mathbf{x}, t) & =\rho(\mathbf{x}, t)^{\gamma-1}, \\
p(\mathbf{x}, t) & =(\gamma-1) \rho(\mathbf{x}, t)^{\gamma},
\end{aligned}
$$

with $\gamma=2, h(t)=\sqrt{1-\frac{t^{2}}{\tau^{2}}}$ and $\tau=\sqrt{\frac{1}{2 \gamma} \frac{R_{2}^{2}-R_{1}^{2}}{\rho_{2}^{\gamma-1}-\rho_{1}^{\gamma-1}}}$. Again, we took $\rho_{1}=1, \rho_{2}=2, R_{1}=0$ and $R_{2}=10$. Final time is set to $t=1.2$.

For this test we considered the initial domain to be $\Omega=] 0,9\left[{ }^{2}\right.$. We took Cartesian grids $10 \times 10,20 \times 20$, $40 \times 40$ and $80 \times 80$ and obtained the convergence rates given in figure 4 .

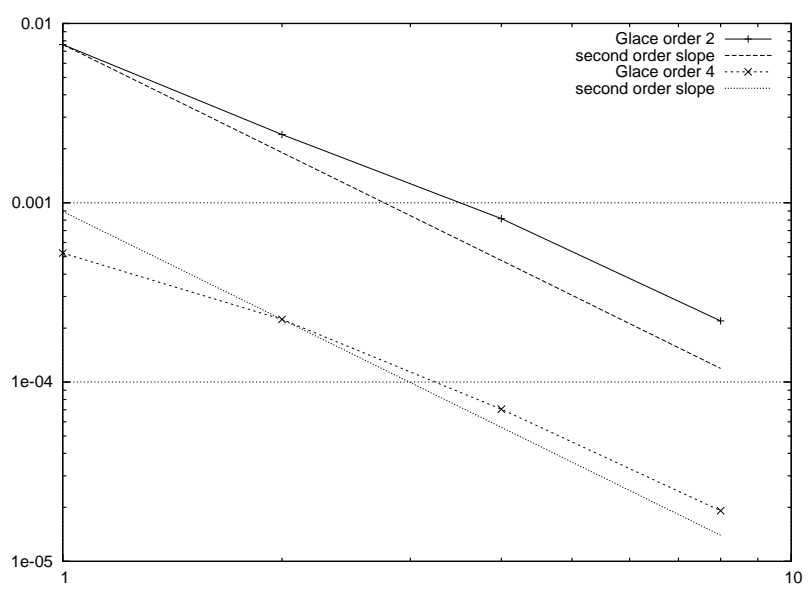

Figure 4. 2D-Kidder isentropic compression on Cartesian grids. Convergence rates for the Glace scheme with RK2-degree 1 and RK4-degree 3 schemes.

This test case is interesting. It shows as expected that the RK4-degree 3 reconstruction scheme is not of order 4 but falls down to second order of accuracy. Moreover, for this particular problem the velocity is linear, so there is no geometrical error by considering only polyhedral cells. The error only comes from the quadrature formulas (8), (10) and (11) which are only exact for $\mathbb{Q}^{1}$ functions, thus of second order.

This test case is ideal for quadrature formulas improvement. 
The nice result is that, even if fourth order is not reached, the RK4-degree 3 reconstruction shows much better accuracy, as we expected at the very beginning of this study.

\subsubsection{Shell implosion test case}

The shell implosion test case (see [5]) is defined as follows. The initial domain is the area between the two circles centered in $\mathbf{0}$ of radius 1 and 0.95 . The initial velocity is $\mathbf{u}(t=0)=-0.74 \frac{\mathbf{x}}{\mathbf{x} \cdot \mathbf{x}}$. The initial density is set to $\rho(t=0)=1$. The initial pressure is $p(t=0)=0.614$ and follows a perfect gas law with $\gamma=1.4$. The final computational time is $t=1$.

This case is interesting with regard to the ICF hydrodynamics. The difficulty of this test is that overdissipative schemes in the ortho-radial direction converges slowly to the solution — see [11] or [5] for more details. The aim of this test is to check if order higher than 1 can cure Chic and Cheng and Shu's schemes.

For this test we measured the internal radius of the shell at time $t=1$ on a computed solution with the order 2 Muscl Glace scheme (see [5]). We used 2000 cells in the radial direction. The obtained minimal radius is 0.0871 . This is our reference solution.

To show that our boundary condition treatment does not degrade symmetries, we used a half computational domain. The chosen mesh is 21 cells in the radial direction and 23 in the ortho-radial direction. Symmetries are preserved with each scheme up to the precision machine. With this coarse mesh, we obtained the results presented in figure 5 .

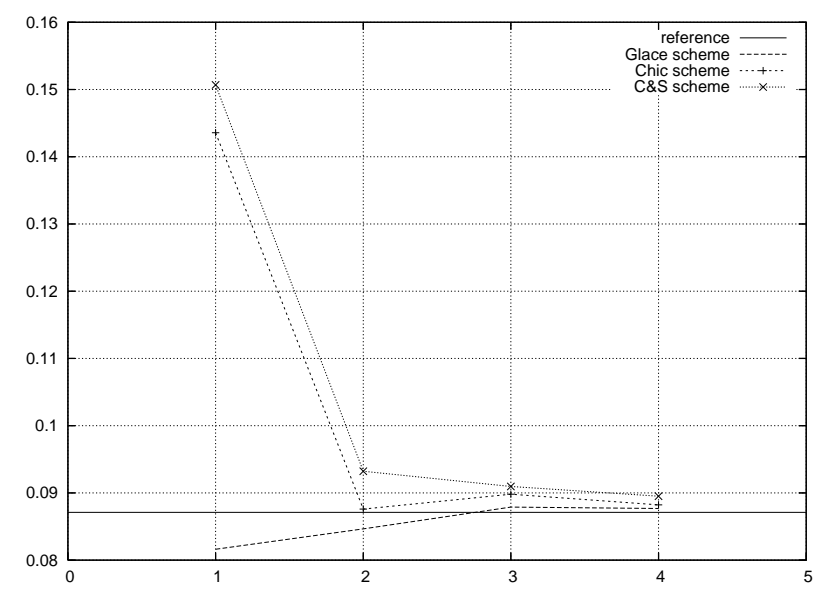

FIGURE 5. Shell implosion test: minimal radius for given orders.

These results clearly show that higher order version of Chic and Cheng and Shu's schemes does not suffer from excessive ortho-radial numerical dissipation. For this test, the limiter was activated.

We finally have to say that we were not able to pass the shell problem test with the Cheng and Shu scheme as is. We had problems imposing the pressure boundary conditions and we removed the tangential contributions of the velocity, replacing formula (22) by

$$
\mathbf{u}_{r}=\frac{1}{2} \sum_{e} u_{e} \mathbf{n}_{e-+} \cdot
$$

\subsection{Non-smooth cases}

Our main interest in this paper is to build a high-order semi-Lagrangian scheme. However, solving Euler equations implies the ability to deal with shocks.

The aim of these tests is to evaluate the limitation strategy developed in section 2.3 . 


\subsubsection{Sod shock tube}

This is the classical Sod shock tube initialized with the two states

$$
\begin{aligned}
\rho(x<0.5, t=0) & =1, & & \rho(x>0.5, t=0)=0.125, \\
u(x, t=0) & =0, & & \\
p(x<0.5, t=0) & =1, & & p(x>0.5, t=0)=0.1 .
\end{aligned}
$$

The pressure follows a perfect gas law and $\gamma=1.4$. The computational domain is $] 0,1[$. Since the simulation is performed using a $2 \mathrm{D}$ code, symmetry conditions are used for the boundaries $y_{\min }$ and $y_{\max }$. For this test, we used 100 cells in the $x$-direction.

The density profiles obtained with the Glace scheme at orders 1 to 4 and the limiter proposed in 2.3 are presented in figure 6 . One can notice the efficiency of the proposed strategy. However, it is not completely

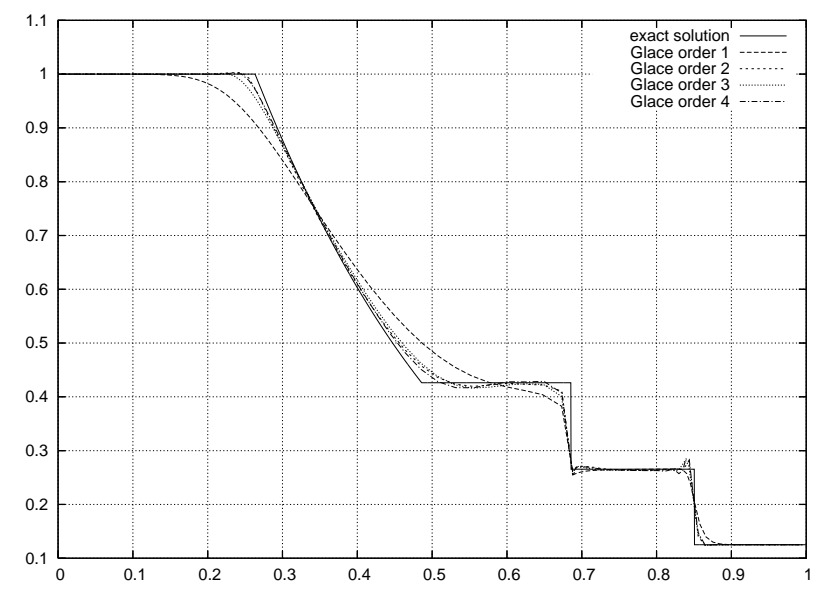

FiguRE 6. Sod shock tube with Glace scheme at orders 1 to 4 compared with the exact solution.

Density profile at time $t=0.2$.

satisfactory since undershoots and overshoots are observed. These are caused by the limitation procedure used for the velocity. Since only the modulus of the velocity is considered, it allows negative values of the velocity fluxes. We observed that if we used a component by component limiter the problem is cured. Nevertheless, for this paper, we kept the multidimensional limiter which preserves symmetries.

\subsubsection{Noh shock tube}

We now present a challenging test case: a cylindrical Noh shock tube using a 2D Cartesian grid. Initial state is

$$
\begin{aligned}
& \rho(t=0)=1, \\
& p(t=0)=10^{-3}, \\
& \mathbf{u}(t=0)=-\frac{\mathbf{x}}{\|\mathbf{x}\|},
\end{aligned}
$$

with $\mathbf{u}(\mathbf{x}=\mathbf{0}, t)=\mathbf{0}$ for all $t>0$ and symmetry conditions for slipping boundaries. The final time for this computation is 0.6. 
One probably has noticed that the initial pressure is not set to $10^{-6}$ as usually. This was the only way to reach final computation time. We believe that this is again due to the proposed limiter which is not restrictive enough for this test. Moreover, we were not able to use better reconstruction as polynomials of degree 2 or 3 . The smaller dissipation of the obtained schemes joined to the bad limitation makes the computation fail.

Nevertheless, we show on figure 7 the results we obtained using the Chic scheme on a $30 \times 30$ grid. The
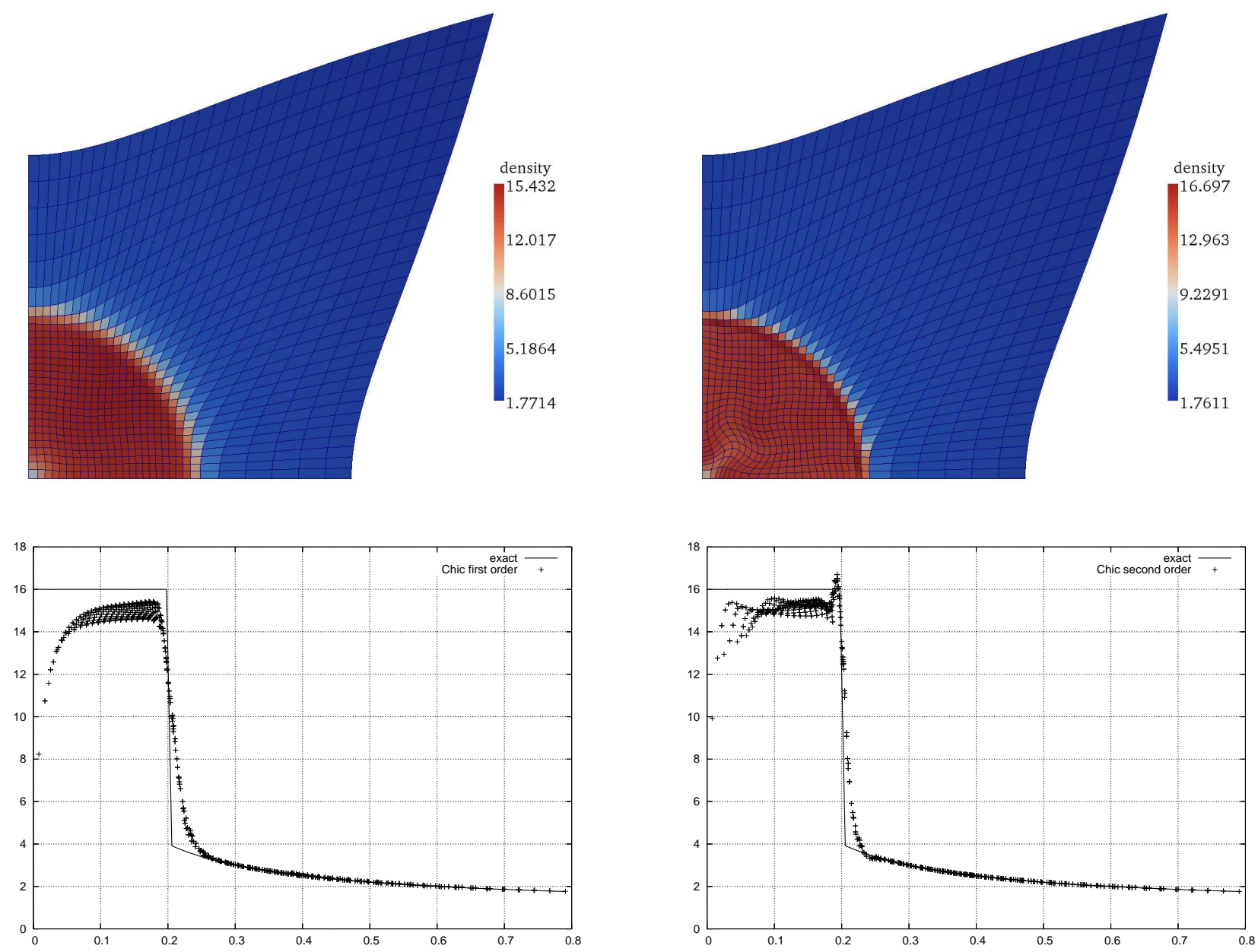

Figure 7. Noh shock tube.

second-order version improves the quality of the result and keeps the symmetry according to the first diagonal.

\section{Conclusions and Perspectives}

In this paper we have studied a way to achieve high-order approximation of Euler equations in semiLagrangian coordinates. It is based on a polynomial reconstruction that preserves symmetries by construction and uses Runge-Kutta for time discretization. The reconstruction method, described in [12], is developed for cell centered schemes, so we used the three different schemes proposed in [7], [9] and [6].

As expected, high-order is only obtained for flows that preserve straight edges of cells and when quadrature is exact. Thus we got the expected orders for $1 \mathrm{D}$ smooth test cases. In addition, we found that the 2D-Kidder problem is a good candidate to validate right quadratures for fluxes computations since there is no geometric 
errors. We also illustrated importance of the choice of the reconstruction variables choice: it is mandatory to consider the conservative set.

Since our purpose was to get high-order schemes, we did not focus on the limiter part but proposed a simplistic strategy. The main feature of this limiter is to preserve symmetries but even if is not restrictive enough it allowed us to deal with some non-regular flows, when shocks are not too strong. So we were not able to use high-order schemes for Noh's problem.

Also, we evaluated the effect of high-order reconstruction to cure excessive ortho-radial dissipation of Chic and Cheng and Shu's schemes. Higher order improves enormously their behavior for the shell problem.

Finally, our evaluation of Cheng and Shu's scheme is the following. First, we had difficulties dealing with boundary conditions. Second, we do not like the compatibility problem for the discretization of the divergence and gradient operators. However, improving the scheme accuracy in using appropriate quadrature for the flux computation is straight away, as described in [6].

The main interest of the current work is to show that achieving high-order semi-Lagrangian scheme is possible. The $\mathbf{C}_{j r}$ formalism allows us to think that the geometric equation (1) can also be approximated with the right order.

However, before considering high-order geometries and high-order quadratures for fluxes computation, it is clear that finding a good limitation strategy is the next step.

The authors address many thanks to Philippe Hoch for his help during the summer school in Lumminy. He helped us to use and modify G0++ [3] to fit our needs. We also thank him for the instructive discussions we had on the topic.

Finally, the authors want to address special thanks to Isabelle Marmajou for her comments and remarks about this document.

\section{REFERENCES}

[1] R. Abgrall. On essentially non-oscillatory schemes on unstructured meshes: analysis and implementation. Journal of Computational Physics, 1994.

[2] R. Abgrall, R. Loubère, and J. Ovadia. A Lagrangian discontinuous Galerkin type method on unstructured meshes to solve hydrodynamics problems. Int. J. Numer. Math. Fluids, 6(44):645-663, 2004.

[3] J. D. Benamou and P. Hoch. GO++: A modular Lagrangian/Eulerians software for Hamilton Jacobi equations of geometric optics type. M2AN, 36(5):883-905, September-October 2002.

[4] D. J. Benson. Computational methods in Lagrangian and Eulerian hydrocodes. Computer Methods in Applied Mechanics and Engineering, 99:235-394, 1992.

[5] G. Carré, S. Del Pino, B. Després, and E. Labourasse. A cell-centered Lagrangian hydrodynamics scheme in arbitrary dimension. submitted to Journal of Computational Physics.

[6] J Cheng and C.-W. Shu. A high order ENO conservative Lagrangian type scheme for the compressible Euler equation. Journal of Computational Physics, 227:1567-1596, 2007.

[7] B. Després and C. Mazeran. Lagrangian gas dynamics in two dimensions and Lagrangian systems. Arch. Rational Mech. Anal., 178:327-372, 2005.

[8] G. H. Golub and C. F. Van Loan. Matrix Computations. John Hopkins University Press, third edition edition, 1996.

[9] P.-H. Maire, R. Abgrall, J. Breil, and J. Ovadia. A cell-centered Lagrangian scheme for two-dimensional compressible flow problems. SIAM Journal of Scientific Computing, 29:1781-1824, 2007.

[10] P.-H. Maire and B. Nkonga. Multi-scale Godunov-type method for cell-centered discrete Lagrangian hydrodynamics. Journal of Computational Physics, In Press, Corrected Proff, 2008.

[11] C. Mazeran. Sur la structure mathématiques et l'approximation numérique de l'hydrodynamique lagrangienne bidimensionnelle. PhD thesis, Université de Bordeaux 1, 2007.

[12] C. F. Ollivier-Gooch. High-order ENO schemes for unstructured meshes based on least-squares reconstruction. Technical report, Mathematics and Computer Science Division, Argonne National Laboratory, 1996.

[13] C. F. Ollivier-Gooch. Quasi-ENO schemes for unstructured meshes based on unlimited data-dependent least-squares reconstruction. Journal of Computational Physics, 1996.

[14] A. van der Sluis. Condition numbers and equilibration of matrices. Numer. Math., 14:14-23, 1969.

[15] J. von Neumann and R. D. Richtmyer. A method for the numerical calculation of hydrodynamics shocks. Journal of Computational Physics, 21:232-237, 1950. 